\title{
Fast Side-Stepping of the Triple Inverted Pendulum via Constrained Nonlinear Feedforward Control Design
}

\author{
Knut Graichen, Michael Treuer, and Michael Zeitz
}

\begin{abstract}
The side-stepping of the triple inverted pendulum serves as a benchmark problem for the presentation of a new feedforward control design technique for finite-time transition problems under consideration of output constraints. The inversion-based design treats the transition task as a twopoint boundary value problem (BVP) defined in the inputoutput coordinates of the pendulum. As a necessary condition for its solvability, a sufficient number of free parameters is provided in a set-up function for the cart acceleration. The new approach allows to directly incorporate output constraints of the pendulum within the BVP, which is solved by a standard MATLAB function. A linear LQR controller is used to stabilize the pendulum along the feedforward side-stepping trajectories. Experimental results for the triple inverted pendulum illustrate the accuracy and robustness of the proposed feedforward/feedback control scheme.
\end{abstract}

\section{INTRODUCTION}

Inverted pendulums are often used in nonlinear control education and research due to challenging features like unstable and nonlinear dynamics as well as nonminimum-phase and nonholonomic behavior. A vast range of contributions exists for the stabilization of different types of inverted pendulums, see e.g. [14], [5], [1]. In the recent past, also the tracking problem has been studied especially for single inverted pendulums [2], [16], [10], [12].

The triple inverted pendulum on a cart is an especially difficult benchmark problem. The control task of stabilizing the pendulum in the upward position has been studied e.g. in [13], [4], [19]. Besides the stabilization aspect, the side-stepping maneuver, i.e. the lateral transition between two unstable upward equilibria, is a challenging tracking control problem. In [15], the flatness-property of the linear pendulum model, which results from the linearization in the upward equilibrium, is used to design a linear feedforward control as part of the two-degree-of-freedom control scheme illustrated in Fig. 1. The feedforward control $\Sigma_{F F}$ solves the side-stepping problem, whereas the feedback control $\Sigma_{F B}$ stabilizes the pendulum along the desired trajectories provided by the signal generator $\Sigma^{*}$.

In this paper, the side-stepping problem with constraints on the cart position, its velocity, and its acceleration as the input to the system is accomplished via nonlinear feedforward control design [6], [7]. The inversion-based feedforward control $\Sigma_{F F}$ treats the side-stepping as a finite-time twopoint boundary value problem (BVP) defined in the inputoutput coordinates of the system. The presented approach allows to directly incorporate the given output constraints within the formulation of the BVP, which is solved in a

K. Graichen, M. Treuer, and M. Zeitz are with Institut für Systemdynamik und Regelungstechnik, Universität Stuttgart, D-70550 Stuttgart, Germany \{graichen, treuer, zeitz\}aisr.uni-stuttgart.de straightforward manner with the standard MATLAB function bvp4c [17]. Due to the accuracy of the nonlinear feedforward control, a simple linear LQR controller is sufficient as the stabilizing feedback part $\Sigma_{F B}$ in Fig. 1. Experimental results reveal the accuracy and robustness of the control scheme.

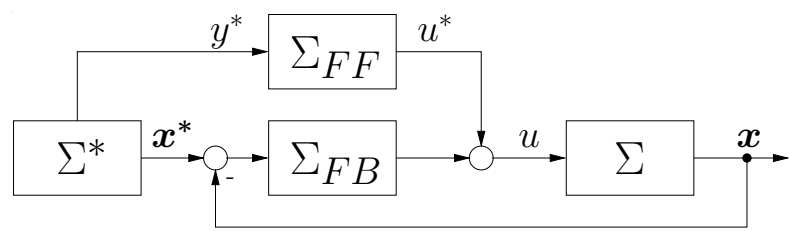

Fig. 1. Two-degree-of-freedom control scheme with system $\Sigma$, feedback control $\Sigma_{F B}$, feedforward control $\Sigma_{F F}$, and signal generator $\Sigma^{*}$.

\section{SIDE-STEPPING MANEUVER OF THE TRIPLE INVERTED PENDULUM}

The triple pendulum in Fig. 2 consists of three links with different lengths $l_{i}$ and the angles $\phi_{i}(t), i=1,2,3$ to the vertical. The first link is attached to a cart moving on a rail with the position coordinate $x_{c}(t)$. The meaning and values of the mechanical parameters are listed in Table I. The acceleration $\ddot{x}_{c}(t)$ of the cart serves as input $u(t)=\ddot{x}_{c}(t)$ to the system. In the experimental set-up (see Section V), $\ddot{x}_{c}(t)$ is controlled by an underlying fast PI-controller. Furthermore, the cart is subject to the following constraints

$$
\begin{aligned}
\left|x_{c}\right| & \leq 0.7 \mathrm{~m} \\
\left|\dot{x}_{c}\right| & \leq 2.2 \mathrm{~m} \mathrm{~s}^{-1} \\
|u|=\left|\ddot{x}_{c}\right| & \leq 20 \mathrm{~m} \mathrm{~s}^{-2}
\end{aligned}
$$

due to the limited rail length and the physical limits of the cart actuator.

\section{A. Equations of motion}

The model of the triple inverted pendulum can be derived via the Lagrange method. Due to the lack of space, the derivation is only shortly described without going into detail. For more explicit information, see e.g. [4]. The absolute position $\boldsymbol{x}^{i}=\left[x_{1}^{i}, x_{2}^{i}\right]^{\mathrm{T}}, i=1,2,3$ of the center of mass of each link $i$ are given by

Link 1: $\quad \boldsymbol{x}^{1}=\left[\begin{array}{c}x_{c}+a_{1} \sin \phi_{1} \\ a_{1} \cos \phi_{1}\end{array}\right]$

Link 2: $\quad \boldsymbol{x}^{2}=\left[\begin{array}{c}x_{c}+l_{1} \sin \phi_{1}+a_{2} \sin \phi_{2} \\ l_{1} \cos \phi_{1}+a 2 \cos \phi_{2}\end{array}\right]$

Link 3: $\quad \boldsymbol{x}^{3}=\left[\begin{array}{c}x_{c}+l_{1} \sin \phi_{1}+l_{2} \sin \phi_{2}+a_{3} \sin \phi_{3} \\ l_{1} \cos \phi_{1}+l_{2} \cos \phi_{2}+a_{3} \cos \phi_{3}\end{array}\right]$. 


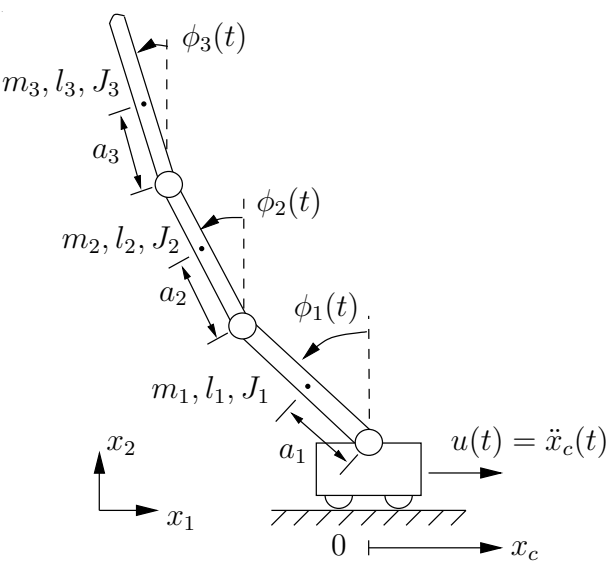

Fig. 2. Schematic of the triple inverted pendulum with the mechanical parameters in Table I.

For the kinetic and potential energy holds:

$$
T=\frac{1}{2} m_{c} \dot{x}_{c}^{2}+\frac{1}{2} \sum_{i=1}^{3}\left[m_{i}\left|\dot{\boldsymbol{x}}^{i}\right|^{2}+J_{i} \dot{\phi}_{i}^{2}\right], \quad V=\sum_{i=1}^{3} m_{i} g x_{2}^{i} .
$$

The non-conservative friction forces in the links are modeled with the linear expressions

$$
\begin{aligned}
& F_{1}=-d_{1} \dot{\phi}_{1}+d_{2}\left(\dot{\phi}_{2}-\dot{\phi}_{1}\right), \\
& F_{2}=d_{2}\left(\dot{\phi}_{1}-\dot{\phi}_{2}\right)+d_{3}\left(\dot{\phi}_{3}-\dot{\phi}_{2}\right), \\
& F_{3}=d_{3}\left(\dot{\phi}_{2}-\dot{\phi}_{3}\right),
\end{aligned}
$$

whereby the parameters $d_{i}$ denote the damping coefficient at the respective link $i$. The Lagrange function $L=T-V$ yields the equations of motion

$$
\frac{\partial}{\partial t} \frac{\partial L}{\partial \dot{\phi}_{i}}-\frac{\partial L}{\partial \phi_{i}}=F_{i}, \quad i=1,2,3 .
$$

Together with the cart dynamics, the overall model of the triple inverted pendulum can be formally written as a system of second-order ODEs

$$
\begin{aligned}
\ddot{x}_{c} & =u \\
\ddot{\boldsymbol{\phi}} & =\boldsymbol{\beta}(\boldsymbol{\phi}, \dot{\boldsymbol{\phi}}, u)
\end{aligned}
$$

with $\phi=\left[\phi_{1}, \phi_{2}, \phi_{3}\right]^{\mathrm{T}}$ and the system order $n=8$. Note that the ODEs (6) are only affected by $u$ and are independent of the cart displacement $x_{c}$ and its velocity $\dot{x}_{c}$.

The side-stepping maneuver within a finite time interval $t \in[0, T]$ requires to move the triple inverted pendulum from the initial equilibrium

$$
x_{c}(0)=x_{0}^{*}, \quad \dot{x}_{c}(0)=0, \quad \phi(0)=\dot{\phi}(0)=0
$$

TABLE I

MECHANICAL PARAMETERS OF THE TRIPLE PENDULUM.

\begin{tabular}{llll} 
Pendulum link & $\begin{array}{l}\text { inner } \\
i=1\end{array}$ & $\begin{array}{l}\text { middle } \\
i=2\end{array}$ & $\begin{array}{l}\text { outer } \\
i=3\end{array}$ \\
\hline length $l_{i}[\mathrm{~m}]$ & 0.323 & 0.419 & 0.480 \\
distance to center of gravity $a_{i}[\mathrm{~m}]$ & 0.2145 & 0.273 & 0.223 \\
mass $m_{i}[\mathrm{~kg}]$ & 0.853 & 0.908 & 0.510 \\
moment of inertia $J_{i}\left[\mathrm{Nms}^{2}\right]$ & 0.0126 & 0.0238 & 0.0164 \\
friction constant $d_{i}\left[\mathrm{Ns} \mathrm{rad}^{-1}\right]$ & 0.005 & 0.005 & 0.005 \\
\hline
\end{tabular}

to the terminal equilibrium

$$
x_{c}(T)=x_{T}^{*}, \quad \dot{x}_{c}(T)=0, \quad \phi(T)=\dot{\phi}(T)=0,
$$

whereby both equilibria are unstable. The total displacement of the cart is $\Delta x^{*}=x_{T}^{*}-x_{0}^{*}$. With respect to the twodegree-of-freedom control scheme in Fig. 1, the feedforward control $\Sigma_{F F}$ has to provide a nominal input trajectory $u^{*}(t)$ which realizes the side-stepping in the open-loop mode.

\section{B. Input-output normal form}

The inversion-based feedforward control $u^{*}(t), t \in[0, T]$ of the side-stepping maneuver is designed in the inputoutput coordinates, cf. [3], [6]. With the cart displacement as the output $y=x_{c}$ and the additional coordinates $\boldsymbol{\eta}=\boldsymbol{\phi}$, the pendulum model (5)-(6) has the relative degree $r=2$, and is already given in the input-output normal form [11]

$$
\begin{aligned}
\ddot{y} & =u \\
\ddot{\boldsymbol{\eta}} & =\boldsymbol{\beta}(\boldsymbol{\eta}, \dot{\boldsymbol{\eta}}, u)
\end{aligned}
$$

with the input-output dynamics (9) and the internal dynamics (10). The zero dynamics of the system following from (10) with $u=0$ is unstable, i.e. the pendulum is nonminimum-phase.

The side-stepping of the pendulum within the finite time interval $t \in[0, T]$ requires the following boundary conditions (BCs)

$$
\begin{array}{lll}
y(0)=x_{0}^{*}, & y(T)=x_{T}^{*}, & \left.\dot{y}\right|_{0, T}=0 . \\
\boldsymbol{\eta}(0)=0, & \boldsymbol{\eta}(T)=0, & \left.\dot{\boldsymbol{\eta}}\right|_{0, T}=0
\end{array}
$$

and the constraints for the output and input

$$
\begin{aligned}
|y| & \leq y_{\max }=0.65 \mathrm{~m} \\
|\dot{y}| & \leq v_{\max }=2 \mathrm{~m} \mathrm{~s}^{-1} \\
|u|=|\ddot{y}| & \leq u_{\max }=15 \mathrm{~m} \mathrm{~s}^{-2}
\end{aligned}
$$

to be satisfied. ${ }^{1}$ The second order ODEs (9)-(10), the BCs (11)-(12), and the constraints (13)-(15) form two coupled nonlinear two-point BVPs for $y(t)$ and $\boldsymbol{\eta}(t)$ in dependence of the input trajectory $u(t), t \in[0, T]$, which is the main objective of the feedforward control design.

\section{FEEDFORWARD CONTROL DESIGN}

The inversion-based design of the feedforward control is based on the inversion of the input-output dynamics [3], [6]. In view of (9), the feedforward control ${ }^{2}$

$$
u^{*}(t)=\ddot{y}^{*}(t)
$$

is simply the second time derivative of the output trajectory $y^{*}(t)$, which must be set-up as a twice differentiable function. In order to determine the trajectory $\boldsymbol{\eta}^{*}(t)$, the BVP of the internal dynamics (10), (12) can be rewritten to

$$
\ddot{\boldsymbol{\eta}}^{*}=\boldsymbol{\beta}\left(\boldsymbol{\eta}^{*}, \dot{\boldsymbol{\eta}}^{*}, \ddot{y}^{*}\right),\left.\quad \boldsymbol{\eta}^{*}\right|_{0, T}=\left.\dot{\boldsymbol{\eta}}^{*}\right|_{0, T}=0
$$

with $\ddot{y}^{*}(t)$ serving as input. Obviously, the BVP is overdetermined with three second-order ODEs and 12 BCs. The

\footnotetext{
${ }^{1}$ The constraints (13)-(15) are stricter compared to the physical limits (1)(3) in order to leave sufficient reserve for the underlying feedback controller.

${ }^{2}$ The asterisk "*” signifies the feedforward variables.
} 
basic idea of the approach presented in [6], [7] is to provide six free parameters $\boldsymbol{p}=\left(p_{1}, \ldots, p_{6}\right)$ in a set-up function $\Upsilon(t, \boldsymbol{p})$ for the output trajectory $y^{*}(t)=\Upsilon(t, \boldsymbol{p})$, as a necessary condition for the solvability of the internal dynamics BVP (17). The set-up $\Upsilon(t, \boldsymbol{p})$ can be constructed e.g. with polynomials or trigonometric functions. The solution of the resulting BVP with free parameters comprises the parameter set $\boldsymbol{p}$ as well as the trajectories $\boldsymbol{\eta}^{*}(t)$ for the link angles of the pendulum. Thereby, the parameter set $\boldsymbol{p}$ determines the shape of the output trajectory $y^{*}(t) .{ }^{3}$ However, this approach only allows to consider the constraints (13)-(15) in a "manual" way, e.g. by increasing the transition time $T$ and rechecking the constraints.

Therefore, this approach has been elaborated in [8], [9] to account for input constraints. In the following, its application to the side-stepping problem of the triple inverted pendulum is shown. In a second step, the remaining output constraints (13)-(14) are incorporated by appropriately adjusting the input constraints.

\section{A. BVPs for constrained input}

In order to directly incorporate the input constraints (15) within the feedforward control design, the feedforward (16) reveals that $u^{*}(t)$ can only be directly influenced by the second time derivative $\ddot{y}^{*}(t)$ of the output. Therefore, a new function $\hat{\alpha}$ is introduced to parametrize $\ddot{y}^{*}(t)=\hat{\alpha}$. This augments the previous BVP (17) of the internal dynamics by the BVP for the output $y^{*}(t)$ :

$$
\begin{aligned}
& \ddot{y}^{*}=\hat{\alpha}, \quad y^{*}(0)=x_{0}^{*}, y^{*}(T)=x_{T}^{*},\left.\dot{y}^{*}\right|_{0, T}=0, \\
& \ddot{\boldsymbol{\eta}}^{*}=\boldsymbol{\beta}\left(\boldsymbol{\eta}^{*}, \dot{\boldsymbol{\eta}}, \hat{\alpha}\right),\left.\quad \boldsymbol{\eta}^{*}\right|_{0, T}=0,\left.\quad \dot{\boldsymbol{\eta}}^{*}\right|_{0, T}=0 .
\end{aligned}
$$

The solutions $y^{*}(t)$ and $\boldsymbol{\eta}^{*}(t)$ of the BVPs (18)-(19) and the feedforward trajectory $u^{*}(t)$ in (16) mainly depend on the set-up of the function $\hat{\alpha}=\ddot{y}^{*}$ with respect to the following objectives:

(i) $\mathcal{C}^{0}$-continuity of the feedforward trajectory $u^{*}(t)$ at the bounds $t=0, T$ implies that the output trajectory $y^{*}(t)$ must meet the two additional $\mathrm{BCs}$

$$
\ddot{y}^{*}(0)=0, \quad \ddot{y}^{*}(T)=0,
$$

which have to be satisfied by the function $\hat{\alpha}=\ddot{y}^{*}(t)$.

(ii) The solvability of the BVPs (18)-(19) defined by four second-order ODEs and $16 \mathrm{BCs}$ requires at least eight free parameters. Similar to the set-up $y^{*}(t)=\Upsilon(t, \boldsymbol{p})$ in [6], [7], the parameters $\boldsymbol{p}=\left(p_{1}, \ldots, p_{8}\right)$ are provided in a function $\Phi(t, \boldsymbol{p})$, which is used to parametrize $\hat{\alpha}=\Phi(t, \boldsymbol{p})$. In the following, a spline set-up is used to construct

$$
\Phi(t, \boldsymbol{p})=\sum_{k=1}^{8} p_{k}\left|t-c_{k}\right|^{3}+\sum_{i=1}^{2} a_{i}(\boldsymbol{p})\left|t-c_{i+8}\right|^{3} .
$$

The free parameters $\boldsymbol{p}=\left(p_{1}, \ldots, p_{8}\right)$ are the coefficients to the spline-function. The two additional coefficients $a_{1}(\boldsymbol{p})$ and $a_{2}(\boldsymbol{p})$ are determined such that the setup function $\Phi(t, \boldsymbol{p})$ satisfies the BCs (20), i.e. $\Phi(0, \boldsymbol{p})=$

\footnotetext{
${ }^{3}$ For instance, $y^{*}(t)=\Upsilon(t, \boldsymbol{p})$ will contain an initial undershoot if the considered system is nonminimum-phase.
}

0 and $\Phi(T, \boldsymbol{p})=0$. The centers $c_{1}, \ldots, c_{10}$ of the cubic basis functions are additional degrees of freedom for the construction of the function $\hat{\alpha}=\Phi(t, \boldsymbol{p}) .{ }^{4}$

(iii) In order to account for the input constraints (15), it is required to check if the resulting feedforward control

$$
u_{\Phi}^{*}=\Phi(t, \boldsymbol{p}),
$$

which follows from (16) with the set-up $\ddot{y}^{*}=\hat{\alpha}=$ $\Phi(t, \boldsymbol{p})$ lies within the constraints (15).

(iv) If $u_{\Phi}^{*}$ is outside the bounds $\pm u_{\max }$, the right-hand side $\hat{\alpha}$ of (18) must be 're-planned' such that the constraints are met. This is accomplished by the following casedependent definition of the function

$$
\hat{\alpha}=\left\{\begin{aligned}
\Phi(t, \boldsymbol{p}) & \text { if }\left|u_{\Phi}^{*}\right| \leq u_{\max } \\
-u_{\max } & \text { if } u_{\Phi}^{*}<-u_{\max } \\
u_{\max } & \text { if } u_{\Phi}^{*}>u_{\max }
\end{aligned}\right.
$$

Note that the set-up $\Phi(t, \boldsymbol{p})$ always determines the function $\hat{\alpha}=\Phi(t, \boldsymbol{p})$ at the interval bounds $t=0, T$, since $u_{\Phi}^{*}(0, \boldsymbol{p})=u_{0}^{*}$ and $u_{\Phi}^{*}(T, \boldsymbol{p})=u_{T}^{*}$ must lie within the interval $\left[u_{\min }, u_{\max }\right]$. Hence, the two additional BCs in (20) are always satisfied by $\Phi(t, \boldsymbol{p})$.

The solution of the BVPs (18)-(19) together with (21)(23) yields the output $y^{*}(t)$ and the internal dynamics state $\boldsymbol{\eta}^{*}(t)$. This concept directly ensures by the case-dependent definition (23) that the input constraints (15) are satisfied [9].

\section{B. Incorporation of output constraints}

In order to incorporate the output constraints (13) and (14), the physical input constraint $u_{\max }$ in (23) is replaced by a variable limit $\hat{u}(y, \dot{y}) \leq u_{\max }$, which is determined in dependence of the output $y$ and the velocity $\dot{y}$ with respect to the constraints (13)-(15) [18]. The new input constraint is used in the case-dependent definition

$$
\hat{\alpha}=\left\{\begin{aligned}
\Phi\left(t, \boldsymbol{p}^{*}\right) & \text { if }\left|u_{\Phi}^{*}\right| \leq \hat{u}(y, \dot{y}) \\
-\hat{u}(y, \dot{y}) & \text { if } u_{\Phi}^{*}<-\hat{u}(y, \dot{y}) \\
\hat{u}(y, \dot{y}) & \text { if } u_{\Phi}^{*}>\hat{u}(y, \dot{y})
\end{aligned}\right.
$$

Thereby, the constraints (13) and (14) for $y$ and $\dot{y}$ are treated separately by calculating a corresponding input limit $u_{1}(y)$ and $u_{2}(\dot{y})$ and defining the respective minimum as the new input constraint:

$$
\hat{u}(y, \dot{y})=\min \left(\hat{u}_{1}(y), \hat{u}_{2}(\dot{y})\right)
$$

Constrained cart position: The limit $\hat{u}_{1}(y)$ accounts for the constraints on the output $y$, namely the cart position $y=$ $x_{c}$. Thereby, $\hat{u}_{1}(y)$ is forced to zero whenever $y$ touches or violates the constraint (13), i.e. $y \geq y_{\max }$. If $y$ stays within a safety region $\Delta y$ away from the physical limit $y_{\max }, \hat{u}_{1}(y)$

\footnotetext{
${ }^{4}$ It is important not to place the centers $c_{9}, c_{10}$ at the boundaries $t=0$ or $t=T$. Otherwise, the spline terms $\left|t-c_{i}\right|^{3}, i=9,10$ would vanish at the respective boundary 0 or $T$, and the coefficients $a_{9}(\boldsymbol{p}), a_{10}(\boldsymbol{p})$ could not be calculated to satisfy the BCs (20).
} 

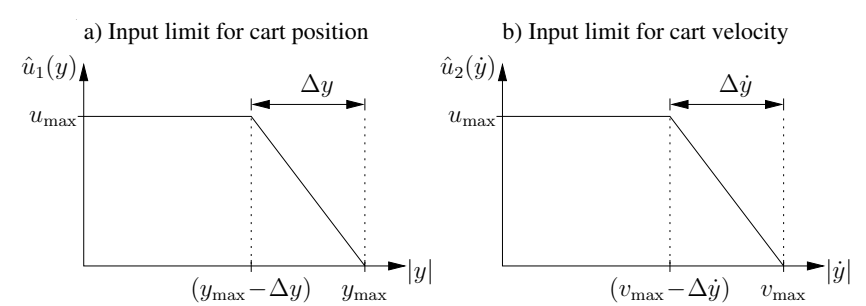

Fig. 3. Ramp functions of the input limits $\hat{u}_{1}(y)$ and $\hat{u}_{2}(\dot{y})$ for the constrained position $|y| \leq y_{\max }$ and velocity $|\dot{y}| \leq v_{\max }$ of the cart.

is set to the maximum acceleration $u_{\max }$. The transition in between is accomplished by the following ramp function:

$$
\hat{u}_{1}(y)=\left\{\begin{array}{cl}
u_{\max } & \text { if }|y| \leq y_{\max }-\Delta y \\
u_{\max } \frac{y_{\max }-|y|}{\Delta y} & \text { if }|y| \in\left(y_{\max }-\Delta y, y_{\max }\right) \\
0 & \text { if }|y| \geq y_{\max }
\end{array}\right.
$$

also see Figure 3a. The ramp function is necessary for a continuous transition between $\hat{u}_{1}(y)=u_{\max }$ and $\hat{u}_{1}(y)=$ 0 , which ensures $\mathcal{C}^{0}$-continuity of the resulting feedforward trajectory $u^{*}(t)$.

The width of the ramp $\Delta y$ is a design parameter, which indirecty affects the jerk necessary to decelerate the cart. It is chosen to $1 \%$ of the maximum allowed position $y_{\max }$.

Constrained cart velocity: In analogy to the cart position, the constraint on the cart velocity (14) is incorporated in the input constraint $\hat{u}_{2}(\dot{y})$ via a ramp function, see Fig. $3 \mathrm{~b}$ ). The input limit $\hat{u}_{2}(\dot{y})$ is zero if $\dot{y}$ touches or violates the constraint (14), i.e. $\dot{y} \geq v_{\max }$. If $\dot{y}$ is smaller than the maximum velocity $v_{\max }, \hat{u}_{2}(\dot{y})$ is increased up to the maximum acceleration $u_{\max }$, i.e.

$$
\bar{u}_{2}(\dot{y})=\left\{\begin{array}{cl}
u_{\max } & \text { if }|\dot{y}| \leq v_{\max }-\Delta v \\
u_{\max } \frac{v_{\max }-|\dot{y}|}{\Delta \dot{y}} & \text { if }|\dot{y}| \in\left(v_{\max }-\Delta v, v_{\max }\right) \\
0 & \text { if }|\dot{y}| \geq v_{\max }
\end{array}\right.
$$

The width of the ramp $\Delta v$ is a second design parameter to control the jerk when the velocity constraint (14) becomes active. It is chosen to $5 \%$ of the maximum allowed velocity $v_{\max }$.

Remark 1: The incorporation of the output constraints (13)-(15) according to (24)-(27) is easy to implement and has the advantage that no further $\mathrm{BCs}$ or inequalities have to be satisfied.

Remark 2: Any output trajectory $y^{*}(t)$ which is a solution of the BVP (18) with (24)-(27) will stay inside the constraints (13) and (14) and will touch them only asymptotically, i.e. $\left|y^{*}(t)\right|<y_{\max }$ and $\left|\dot{y}^{*}(t)\right|<v_{\max }$. This can be explained by contradiction: assume that the output constraint (13) or (14) is violated at the time instant $t=\bar{t} \in(0, T)$. The definitions (26) and (27) ensure that the input constraint $\hat{u}(y, \dot{y})$ is set to zero, which leads to $\ddot{y}(\bar{t})=0$ in (18). Hence, $\dot{y}(t)$ is constant and $y(t)$ will follow a ramp profile, such that the respective constraint (13) or (14) remains violated and $\ddot{y}(t)=0$ holds for all time $t \geq \bar{t}$. In this case however, the BCs in (18) for $t=T$ cannot be met and no solution exists for the BVP.

\section{Numerical SOlution OF THE BVPS}

The numerical solution of the nonlinear BVPs (18)-(19) with (21) and (24)-(27) including the set of free parameters $\boldsymbol{p}=\left(p_{1}^{*}, p_{2}^{*}, \ldots, p_{8}^{*}\right)$ is a standard task in numerics and is solved with a standard MATLAB function.

\section{A. The MatLaB function bvp4c}

The bvp4c-function [17] is a finite-difference code and determines a numerical solution by solving a system of algebraic equations resulting from the difference approximation. Moreover, bvp $4 \mathrm{c}$ estimates the error of the numerical solution on each subinterval and adapts the mesh points. The user must provide the initial points of the mesh as well as a guess for the solution at the mesh points. Furthermore, an initial guess of the free parameters of the BVP is needed.

A linear interpolation between the corresponding BCs on a uniform mesh with 50 grid points $t_{k} \in[0, T]$ serves as a reasonable guess for the trajectories $\boldsymbol{\eta}^{*}\left(t_{k}\right)$ and $y^{*}\left(t_{k}\right)$. The initial guess for the unknown parameters is $\boldsymbol{p}=\mathbf{0}$. For a faster and more robust numerical solution, the analytical Jacobian matrix of the ODEs (18)-(19) is provided to the bvp4c-function.

\section{B. Distribution of the spline centers}

The centers $c_{1}, \ldots, c_{10}$ of the spline set-up $\Phi(t, \boldsymbol{p})$ in (21) are additional design parameters and can be condensed individually in a region where more "activity" of the setup function $\Phi(t, \boldsymbol{p})$ is required. Therefore, the centers $c_{i}$ are mapped over the time interval $t \in[0, T]$ in dependence of a stretching factor $\kappa \geq 1$ :

$$
c_{k}= \begin{cases}T / 2\left(1-(1.1-0.2 k)^{1 / \kappa}\right) & k=1, \ldots, 4 \\ T / 2\left(1+(0.2 k-0.7)^{1 / \kappa}\right) & k=5, \ldots, 8 \\ T / 2\left(1-(0.1)^{1 / \kappa}\right) & k=9 \\ T / 2\left(1+(0.1)^{1 / \kappa}\right) & k=10\end{cases}
$$

For $\kappa=1$, the centers $c_{i}$ are placed uniformly over the time interval $t \in[0, T]$, whereas an increasing $\kappa$ moves the centers $c_{i}$ closer to the boundaries $t=0$ and $t=T$ while symmetry to the interval center $t=T / 2$ is retained. The distribution of the centers in dependence of $\kappa$ is illustrated in Fig. 4, where also two spline basis functions $\left|t-c_{i}\right|^{3}$ are depicted. The centers $c_{9}$ and $c_{10}$ corresponding to the coefficients $a_{1}(\boldsymbol{p})$ and $a_{2}(\boldsymbol{p})$ in (21) are chosen closest to the middle of the time interval to keep the calculation of $a_{1}(\boldsymbol{p})$

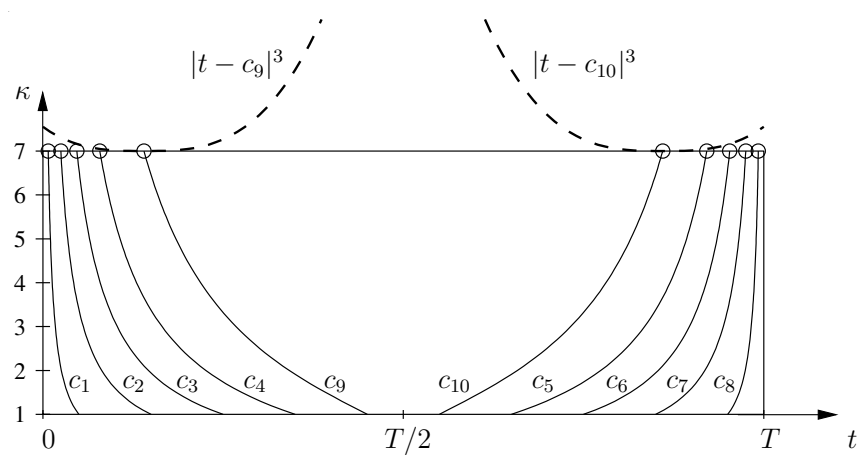

Fig. 4. Placement of the spline centers $c_{i}$ with respect to the design parameter $\kappa$. Two of the spline basis functions $\left|t-c_{i}\right|^{3}$ are shown schematically with dashed lines. 
and $a_{2}(\boldsymbol{p})$ well conditioned. In the following, the stretching factor is set to $\kappa=7$. The condensing of the centers $c_{i}$ at the interval boundaries accounts for the fact that a high acceleration of the cart is especially required at the beginning and the end of the side-stepping.

\section{Simulation results}

Figure 5 shows the computed trajectories $y^{*}(t), \dot{y}^{*}(t)$, $\boldsymbol{\eta}^{*}(t)$ and $u^{*}(t)$ for a side-stepping maneuver over the distance $\Delta x^{*}=1 \mathrm{~m}$ (setpoints $x_{0}^{*}=-0.5 \mathrm{~m}$ and $x_{T}^{*}=$ $0.5 \mathrm{~m}$ ) for the transition time $T=2.5 \mathrm{~s}$. In order to achieve this fast transition, the output $y$ and the velocity $\dot{y}$ of the cart touch the limits (13) and (14). In these time intervals, the set-up function $\hat{\alpha}$ in (24) is determined by $\hat{\alpha}= \pm \hat{u}(y, \dot{y})$ instead of $\Phi(t, \boldsymbol{p})$.

The constraints are also illustrated by the time-discrete snapshots of the side-stepping maneuver in Fig. 6. During the time interval $t \in[0.6 \mathrm{~s}, 0.8 \mathrm{~s}]$ at the beginning of the transition, the cart "waits" at the limit $-y_{\max }$ until the three pendulum links turn in the direction of the side-stepping. During the transition, the cart moves with maximum velocity $v_{\max }$ and finally "waits" again for the pendulum links at the other maximum position $y_{\max }$.

The feedforward trajectory $\ddot{y}^{*}=u^{*}(t)$ illustrates the potential of the set-up $\Phi(t, \boldsymbol{p})$ in (21). The distribution (28) of the spline centers $c_{i}$ closer to the boundaries $t=0$ and $t=T$ allows a more aggressive acceleration and deceleration of the cart at the beginning and the end of the side-stepping maneuver.

Remark 3: As pointed out in Remark 2, the trajectories $y^{*}(t)$ and $\dot{y}^{*}(t)$ do not exactly touch the constraints (13) and (14). However, the results in Fig. 5 clearly show that this effect is negligible, since $y^{*}(t)$ and $\dot{y}^{*}(t)$ approach the limits $y_{\max }$ and $v_{\max }$ in an asymptotic but aggressive manner.
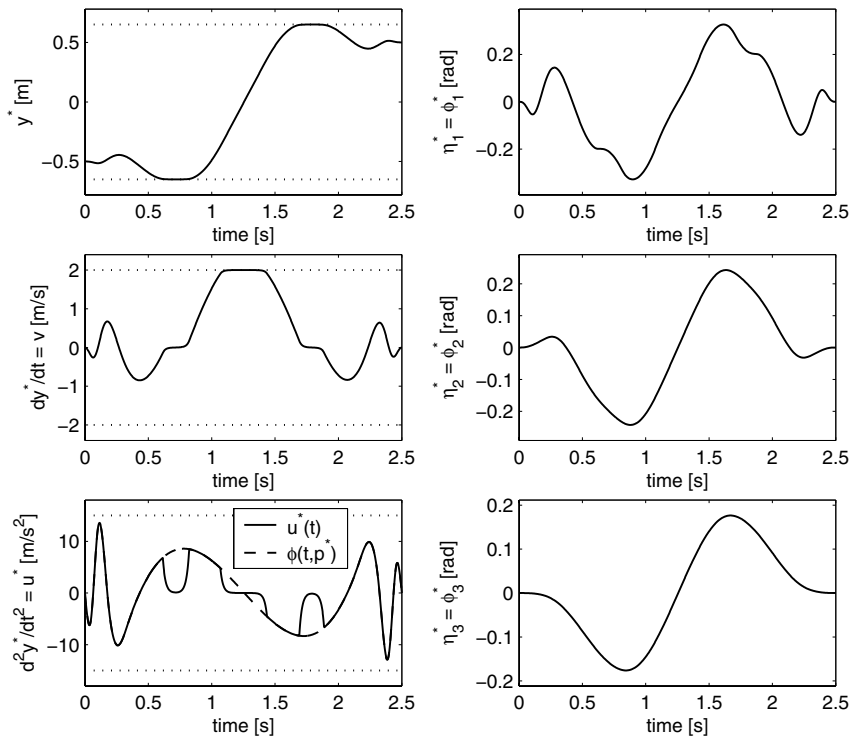

Fig. 5. Nominal feedforward trajectories $\left|y^{*}(t)\right| \leq y_{\max },\left|\dot{y}^{*}(t)\right| \leq v_{\max }$, $\left|u^{*}(t)\right| \leq u_{\max }$ and $\eta^{*}(t)$ for a transition time $\bar{T}=2.5 \mathrm{~s}$.

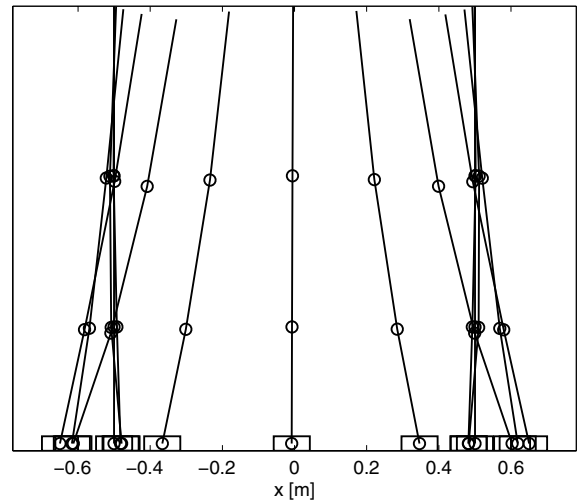

Fig. 6. Time-discrete snapshots of the side-stepping maneuver by the nominal trajectories in Fig. 5.

\section{EXPERIMENTAL RESULTS}

The experimental implementation of the side-stepping requires a stabilization of the triple inverted pendulum by a feedback controller $\Sigma_{F B}$, see Fig. 1. Due to the accuracy of the nonlinear feedforward control, the feedback part $\Sigma_{F B}$ is designed as an LQR controller with the pendulum model linearized in the upper unstable equilibrium, see [15]. Thereby, the linearized model is dynamically extended by one state, in order to compensate for a possible steady state error in the cart position $x_{c}$. For the implementation of the state feedback controller, a Luenberger observer based on the nonlinear model (5)-(6) is used to estimate the state. The error dynamics of the observer is designed by eigenvalue assignment based on the linearized pendulum model.

The tracking control has been validated in experiments with the triple inverted pendulum corresponding to the model parameters in Table I. The controller algorithm is implemented on a $933 \mathrm{MHz}$ computer running under realtime Linux with a sampling time of $1 \mathrm{~ms}$. The incremental angle encoders have a resolution of $2 \pi / 8192 \mathrm{rad}$ and transmit their information through optical links in the joints to reduce friction. The cart is driven by a toothed belt connected to a synchronous motor. The nominal trajectories $y^{*}(t)=x_{c}^{*}(t)$, $\boldsymbol{\eta}^{*}(t)=\phi^{*}(t)$, and the feedforward control $u^{*}(t)$ are calculated offline and stored in look-up tables.

Figure 7 shows the experimental and nominal trajectories of the angles $\phi_{i}(t), i=1,2,3$, the position $y(t)=x_{c}(t)$ and velocity $\dot{y}(t)=\dot{x}_{c}(t)$ as well as the input $u(t)$ for the side-stepping of $\Delta x^{*}=1 \mathrm{~m}$ in the transition time $T=2.5 \mathrm{~s}$. The profiles reveal the good tracking of the triple inverted pendulum. The deviations of the input $u(t)$ in closed-loop compared to the feedforward control $u^{*}(t)$ are mainly due to the response time of the underlying PI controller for the cart acceleration $u=\ddot{y}$, which has to deal with the aggressive feedforward control $u^{*}(t)$ for the sidestepping in $T=2.5 \mathrm{~s}$. Furthermore, the stabilization of the triple pendulum in the upward position leads to a permanent oscillation of approximately $\pm 2 \mathrm{~m} / \mathrm{s}^{2}$.

Fig. 8 shows the nominal and experimental trajectories for the same transition with the larger side-stepping time $T=3 \mathrm{~s}$. The nominal trajectories $y^{*}(t)=x_{c}^{*}(t), \boldsymbol{\eta}^{*}(t)=$ $\phi^{*}(t)$, and the feedforward control $u^{*}(t)$ do not touch the 

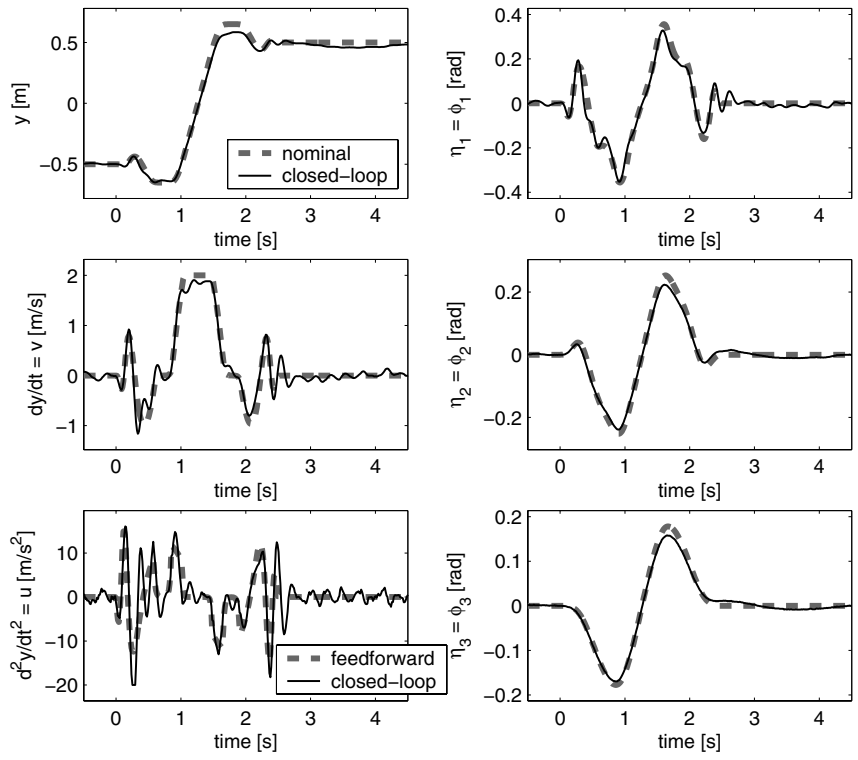

Fig. 7. Experimental and nominal trajectories of the angles $\phi_{i}, i=1,2,3$, the cart position $y=x$, the cart velocity $\dot{y}=v$ and the input $u$ for a sidestepping of $\Delta x^{*}=1 \mathrm{~m}$ in the transition time $T=2.5 \mathrm{~s}$.

physical constraints. Since the profiles are not as aggressive as in Fig. 7 for the transition time $T=2.5 \mathrm{~s}$, the tracking performance is very good and the measured trajectories are close to the nominal ones.

\section{CONCLUSIONS}

The presented design approach of nonlinear feedforward control treats the side-stepping of the triple inverted pendulum as a two-point BVP with free parameters, which can be solved in straightforward manner. The input constraints are exactly met by case-dependently re-planning the setup function for the cart acceleration [8], [9]. This has the advantage that the constraints are directly incorporated into the formulation and solution of the BVPs. The concept also allows to further account for the constraints on the position and velocity of the cart by appropriately reducing the input constraints below the physical limit and setting them to zero if the constraints are violated.

Open questions of the applied two-degree-of-freedom control scheme with a nonlinear feedforward and a linear feedback part concern the existence and uniqueness of solutions to the BVP with output constraints in course of the feedforward design.

\section{ACKNOWLEDGMENTS}

The authors gratefully acknowledge the valuable discussions with Torsten Nutsch and his support with the experimental setup of the triple inverted pendulum.

\section{REFERENCES}

[1] M.J. Anderson and W.J. Grantham, Lyapunov optimal feedback control of a nonlinear inverted pendulum, ASME J. Dyn. Syst. Meas. Contr., vol. 111, 1989, pp. 554-548.

[2] S. Devasia, Stable inversion for nonlinear systems with nonhyperbolic internal dynamics, in Proc. 36th Conf. Dec. Contr., 1997, pp. 28822888.

[3] S. Devasia, D. Chen, and B. Paden, Nonlinear inversion-based output tracking, IEEE Trans. Automat. Contr., vol. 41, 1996, pp. 930-942.
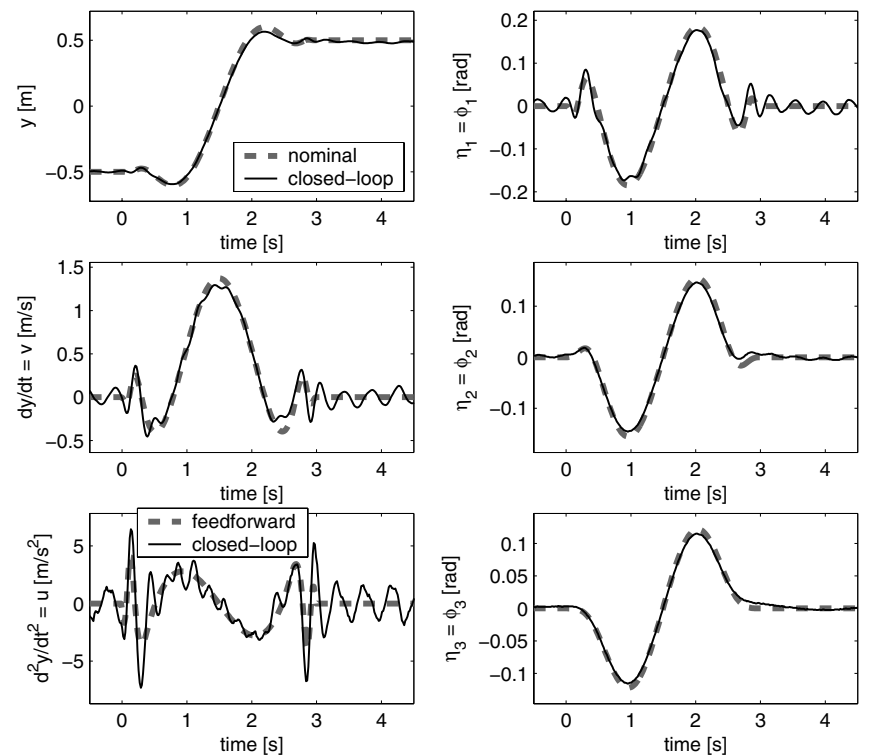

Fig. 8. Experimental and nominal trajectories of the angles $\phi_{i}, i=1,2,3$, the cart position $y$, the cart velocity $\dot{y}=v$ and the input $u$ for a sidestepping of $\Delta x^{*}=1 \mathrm{~m}$ in the transition time $T=3 \mathrm{~s}$.

[4] K.G. Eltohamy and C.-Y. Kuo, Nonlinear optimal control of a triple inverted pendulum with single control input, Int. J. Contr., vol. 69, 1998, pp. 239-256.

[5] K. Furuta, H. Kajiwara, and K. Kosuge, Digital control of a double inverted pendulum on an inclined rail, Int. J. Contr., vol. 32, 1980, pp. 907-924.

[6] K. Graichen, V. Hagenmeyer, and M. Zeitz, A new approach to inversion-based feedforward control design for nonlinear systems, Automatica, scheduled for 41(12), 2005.

[7] K. Graichen, V. Hagenmeyer, and M. Zeitz, Van de Vusse CSTR as a benchmark problem for nonlinear feedforward control design techniques, in Preprints NOLCOS, Stuttgart/D, 2004, pp. 1415-1420.

[8] K. Graichen and M. Zeitz, Nonlinear feedforward and feedback tracking control with input constraints solving the pendubot swing-up problem, Preprints IFAC World Congress, Prague/CZ, 2005.

[9] K. Graichen and M. Zeitz, Feedforward control design for nonlinear systems under input constraints, in T. Meurer, K. Graichen, and E.D. Gilles (eds.): Control and Observer Design for Nonlinear Finite and Infinite Dimensional Systems, LNCIS 322, pp. 235-252. Springer, 2005.

[10] J. Huang, Asymptotic tracking of a nonminimum-phase nonlinear system with nonhyperbolic zero dynamics, IEEE Trans. Automat. Contr., vol. 45, 2000, pp. 542-546.

[11] A. Isidori, Nonlinear Control Systems, Springer, 3rd edition, 1995.

[12] F. Mazenc and S. Bowong, Tracking trajectories of the cart-pendulum system, Automatica, vol. 39, 2003, pp. 677-684.

[13] G.A. Medrano-Cerda, Robust stabilization of a triple inverted pendulum-cart, Int. J. Contr., vol. 68, 1997, pp. 849-865.

[14] S. Mori, H. Nishihara, and K. Furuta, Control of unstable mechanical system - Control of pendulum, Int. J. Contr., vol. 23, 1976, pp. 673692.

[15] T. Nutsch and V. Hagenmeyer, Two-degree-of-freedom control scheme with state feedback applied to a triple inverted pendulum, submitted, 2005.

[16] L.E. Ramos, B. Castillo-Toledo, and S. Negrete, Nonlinear regulation of a seesaw-inverted pendulum, in Proc. IEEE Int. Conf. Contr. Appl., 1998, pp. 1399-1403.

[17] L.F. Shampine, J. Kierzenka, and M.W. Reichelt, Solving boundary value problems for ordinary differential equations in MATLAB with bvp4c, 2000, ftp://ftp.mathworks.com/pub/doc/papers/bvp/

[18] M. Treuer. Entwurf von Vorsteuerungen mit Beschränkungen für Mehrfachpendel (in German). Diploma Thesis (unpublished), Universität Stuttgart, Germany, 2005.

[19] V.A. Tsachouridis and G.A. Medrano-Cerda, Discrete-time $H_{\infty}$ control of a triple inverted pendulum with single control input, IEE Proc. Contr. Theory Appl., vol. 146, 1999, pp. 567-577. 